\title{
Dental Inclination and Thickness of The Alveolar Bone Around Incisors and Molars According to the Vertical facial Tupe
}

\author{
Dr Ahia Monique Lydie BEUGRE-KOUASSI,' Dr Boimyo Eric KOFFI,² Dr Jean-Bertin BEUGRE³ \\ 'Assistant Professor, ${ }^{2}$ Post graduate student, ${ }^{3}$ Professor
}

Department of Orthodontics, Faculty of Dentistry, Felix Houphouet-Boigny University, Abidjan, Côte d'Ivoire.

Correspondence: Dr Ahia Monique Lydie BEUGRE-KOUASSI; Email: ahiakoufr@yahoo.fr

\section{ABSTRACT}

Introduction: The teeth are incline differently according to the vertical skeletal typology and the corresponding alveolar bones do not always have the same thickness. However, the conflicting results in the literature, mainly related to the classification of patients according to vertical characteristics, creates a challenge for the clinician in determining the characteristic inclinations of teeth in a specific patient. The objective of this study was to find out dental inclination and thickness of the alveolar bone around incisors and molars in different facial type.

Materials \& Method: The sample included 51 CBCT radiographs (28 females and 23 males) and subdivided into three subgroups according to vertical facial skeletal pattern (22 normodivergent subjects, 06 hypodivergent subjects and 23 hyperdivergent subjects). Reformatted $\mathrm{CBCT}$ images were used to measure the thickness of the vestibular and lingual alveolar bone around the incisors and first molars at two levels of root length (apical and middle) and their inclination. The Kruskal-Wallis test for the comparison of more than 2 means was applied for statistical analysis.

Result: Alveolar bone thickness in the apical region of the lingual side in hyperdivergent subjects was thicker than in the other two groups. The bone wall is thicker in the lingual than in the vestibular, regardless of the vertical skeletal typology. These varied inclinations were more pronounced anterior sector.

Conclusion: Dental inclinations have close relationships with the alveolar bone thicknesses associated with them. The apex of the root of the lower incisor is closer to the lingual alveolar crest when it was inclined in the lingual direction. There is a significant association between bone thickness around the teeth and facial skeletal divergence.

Keywords: Alveolar bone thickness, Cone Beam, Tooth inclination, Vertical facial type.

\section{INTRODUCTION}

The usual clinical results searched by orthodontic treatments are architectural harmonization of the jaws and their dental arches, resulting in a facial aesthetic modification. This aesthetic impact is essential for both the patient and the practitioner. But, in addition to aesthetic, periodontal health and the alveolar bone limits are important factors in orthodontic treatment. Age, gender and ethnic group are also important in establishing an appropriate orthodontic treatment plan; another important factor is the facial growth pattern and its several clinical characteristics. ' Like the sagittal jaw relationships, vertical growth affects the thickness of the supporting bone. ${ }^{2}$ Patients with long face usually have less supporting bones, which reduces the allowed movement available for teeth before reaching the anatomical limits.

In addition to the thickness of the alveolar bone, dental inclinations must be appropriate to ensure functional and aesthetic occlusion. The inclination of the teeth remains one of the six keys to Andrew's normal occlusion. ${ }^{3}$ It is important not only for occlusal intercuspation but also for the aesthetics of the frontal smile. ${ }^{4}$

Studies have shown variable results with regard to the buccolingual inclination of teeth with respect to the type of vertical growth. ${ }^{5-7}$ Some authors have found that posterior teeth in hypodivergent patients were more lingually inclined than in hyperdivergent subjects. ${ }^{2}$ Masumoto et $\mathrm{al}^{6}$ found in subjects with normal occlusion that hypodivergent subjects had second molars more lingually inclined than hyperdivergent subjects. Grosso et al, ${ }^{7}$ who classified subjects only by vertical facial type, demonstrated that the maxillary and mandibular molars of the hyperdivergent group were lingually inclined.

The heterogeneity of the results in the literature, mainly related to the classification of patients according to sagittal or vertical characteristics, creates a challenge for the clinician in determining the characteristic inclinations of teeth in a specific patient. ${ }^{8}$ 
However, the information provided by conventional radiographic cephalometry is limited by its twodimensional (2D) nature with inaccuracy due to the deformation of anatomical structures. To overcome this disadvantage, CBCT offers the possibility of accessing volumetric data, which allows the morphology of the alveolar-dental complex and the 3D orientation of the teeth to be evaluated.

Hence the interest of our study, the objective of which was to evaluate tooth inclinations and alveolar bone thickness in black African subjects with the CBCT according to vertical facial type.

\section{MATERIALS AND METHOD}

Following IRB approval (\# 000111917 ) by the National Research Ethics Committee, 500 pretreatment CBCT scans of black African subjects were collected from the archive of a private dental practice specializing in Orthodontics and Oral surgery. The scans were performed for other reasons and not specifically for this study. The collected scans belonged to patients aged between 18 and 50 years old. All the subjects were scanned using the same with the same equipment: CS 8100 3D CBCT (Carestream Dental LLC, Atlanta, GA), with the same exposure settings $(8 \mathrm{~mA}, 90 \mathrm{kV}$, voxel

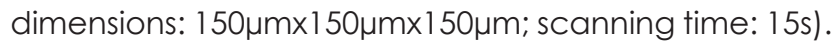
All CBCT images provided a slice thickness of $0.25 \mathrm{~mm}$. Only scan imagery with a field of view (FOV) including the complete dentition of the two artefact-free arches were included.

The other criteria of inclusion patients were, no previous orthodontic treatment; no prior craniofacial trauma, surgery, or symptoms of TMJ joints disorders; a permanent and full dentition with a nearly normal occlusion. The criteria for non-inclusion were, prosthetic crowns or major restorations on the teeth or severe tooth wear; a systemic and disfiguring disease; a very aggressive periodontal disease. After the application of our inclusion and non-inclusion criteria the study sample consisted of 51 subjects ( 28 females and 23 males).

The data from the 3D Scanner X-rays were recorded in DICOM (Digital Imaging and Communication in Medicine) format and processed using SC 3G Imaging (Carestream ${ }^{\circledR}$ ) software.

Subject were divided into three groups according to facial profile, 22 normodivergent subjects, 06 hypodivergent and 23 hyperdivergent subjects. Due to the reduced acquisition fields, which do not allow the base of the skull to be visualized, we used the facial index (IF). This is the ratio of posterior facial height to lower anterior facial height that Horn9 in 1992 named facial index which is normally equal to 0.7. A lower facial index (IF) indicates facial hyperdivergence.

The CBCT images were oriented along the Frankfort Horizontal Plane (FHP) and the median sagittal plane and the bispinal plane and the perpendicular to the FHP and mediosagittal plane.

The images were oriented in relation to the long axis of the tooth (Fig 1) and displayed in coronal slices. Measurements at the mandible and maxilla were performed in the same way.

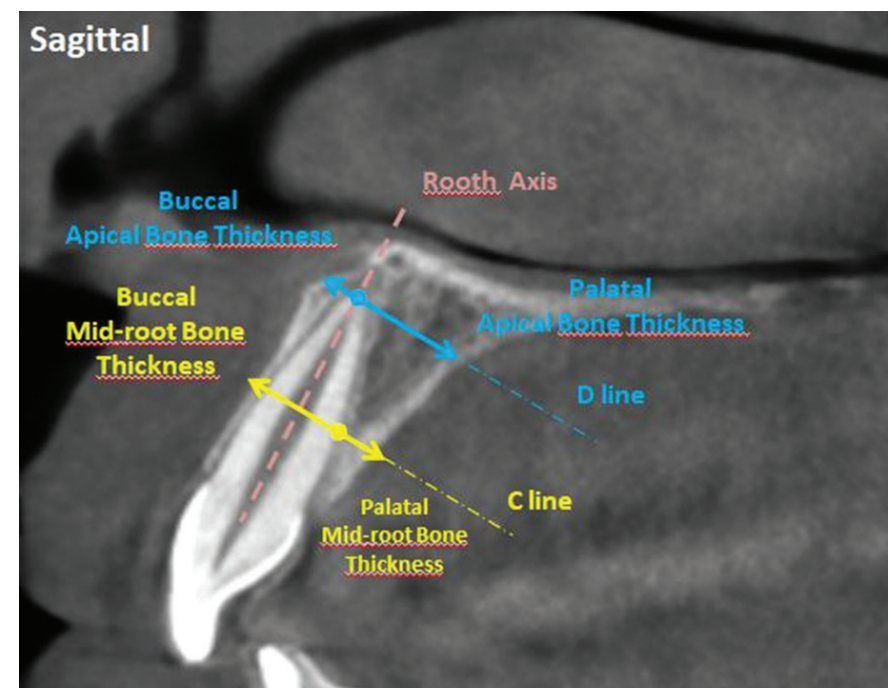

Figure 1: Thickness of the alveolar bone in the buccal and lingual regions at $C$ and $D$ lline on the incisors

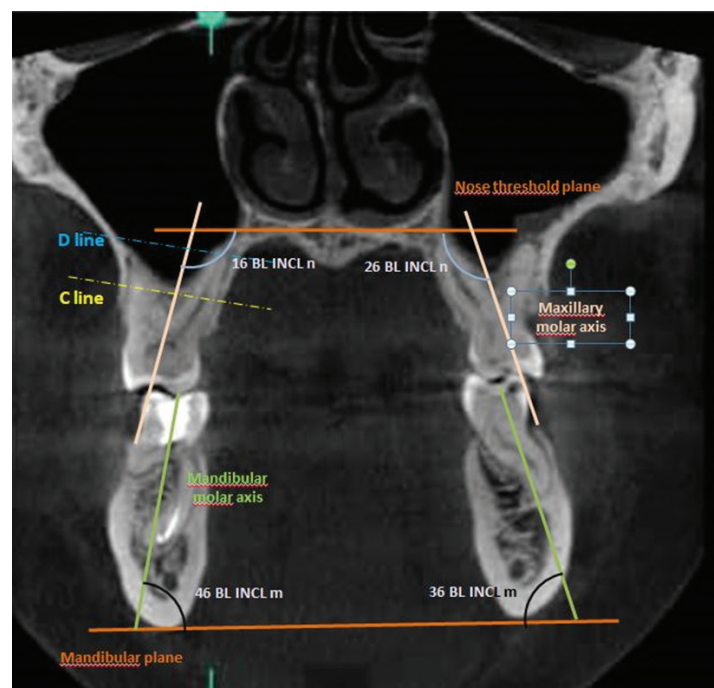

Figure 2: Buccolingual inclination of the molars 
Table 1: Definitions of measurement used in this study

\begin{tabular}{|l|l|}
\hline Measurement variables & \multicolumn{1}{c|}{ Definition } \\
\hline Max CLV & Thickness of the vestibular alveolar bone at C Line at the maxilla \\
\hline Max CLP & Thickness of the lingual alveolar bone in C Line at the maxilla \\
\hline Man CLV & Thickness of the vestibular alveolar bone at C Line at the mandible \\
\hline Man CLL & Thickness of the lingual alveolar bone at C Line at the mandible \\
\hline Max DLV & Thickness of the vestibular alveolar bone at $D$ Line at the maxilla \\
\hline Max DLP & Thickness of the lingual alveolar bone in D Line at the maxilla \\
\hline Man DLV & Thickness of the vestibular alveolar bone at D Line at the mandible \\
\hline Man DLL & Thickness of the lingual alveolar bone at D Line at the mandible \\
\hline MD INCL & Mesiodistal inclination of the incisor with respect to the median sagittal plane \\
\hline BL INCL $n$ & Buccolingual inclination of the molar with respect to the nose threshold plane \\
\hline BL INCL $m$ & Mesiodistal inclination of the molar with respect to the mandibular plane \\
\hline Axis INCL $m$ & Inclination of the axis of the tooth relative to the mandibular plane \\
\hline Axis INCL $n$ & Inclination of the axis of the tooth with respect to the plane of the nose threshold \\
\hline Axix INCL $p$ & Inclination of the axis of the tooth with respect to the palatal plane \\
\hline I/i & Inter-incisal angle formed by the axis (I) of the central incisor and the axis (i) of the lower central incisor. \\
\hline
\end{tabular}

With regard to the thickness of the alveolar bone, we considered four measurements from the cementoenamel junction (CEJ) along 2 reference lines, namely line $\mathrm{C}$ at half the distance of the cementoenamel and apex junction of the tooth and line $D$ at the apex of the tooth:

- $\quad$ Line C (LC) at $1 / 2$ from the distance CEJ - Apex of the tooth

- $\quad$ Line D (LD) at the apex of the tooth (Fig 1)

These measurements involved the incisors and first molars and were carried out on both reference lines, on the buccal side as well as on the lingual side.

We examined incisors inclinations with respect to the palatal plane, the mandibular plane and the median sagittal plane and the interincisal angle.

For molars, we considered their angulation in relation to the plane of the nose threshold, the mandibular plane and the palatal plane. (Fig 2)

in order to differentiate the variables according to the tooth, the number of the tooth concerned was added as a prefix to the variable concerning the thickness of the alveolar bone (e.g.: 11 Max CLV = thickness of the vestibular alveolar bone at the level of 11 at the reference $\mathrm{C}$ line), (Table 1$)$

\section{Statistical analysis}

All measurements were performed by the same operator (K.B.E.). The data from the measurements generated with SC 3D Imaging were statistically analyzed using SPSS (Statistical Package for Social Sciences) 22.0 for Windows (IBM®). We used the normality test (Kolmogorov-Smirnov) to verify the normality of the distribution of the variables studied. Fifteen randomly selected subjects were re-measured by the same examiner (K.B.E.) after 2 weeks to verify intra-examination reproducibility. A paired t-test was performed to verify the existence of significant differences from the initial measurements.

The data were expressed as a mean and standard deviation. The Levene test was used to determine the equality of variances between samples. Finally, the Kruskal-Wallis non-parametric test was used. We considered a statistical level of signification of $5 \%$.

\section{RESULT}

The sample included 51 untreated adult subjects, including 23 males (mean age 28.63 years \pm 11.99 years) and 28 females (mean age 29.72 years \pm 11.47 years) who met the inclusion criteria. No statistically significant differences ( $p=0.569$ ) were observed between the ages of the two gender.

The teeth are tilted differently according to the vertical skeletal typology. The various inclinations were more pronounced in the anterior sector (Table 2). The is no significant difference in dental inclination of the first molars according to the vertical facial profile. The bone wall is thicker in the lingual than in the vestibular, regardless of the vertical skeletal typology (Table 3). 
Table 2: Incisor inclination values (degree) according to vertical facial type

\begin{tabular}{|c|c|c|c|c|}
\hline Tooth inclination & Normodivergent $(n=22)$ & Hypodivergent $(n=6)$ & Hyperdivergent $(n=23)$ & Kruskal Wallis ( $p$-value) \\
\hline $11 \mathrm{MD}$ INCL & $2.90 \pm 1.90$ & $2.33 \pm 1.63$ & $4.00 \pm 3.66$ & NS \\
\hline $12 \mathrm{MD}$ INCL & $4.09 \pm 2.50$ & $3.16 \pm 2.92$ & $4.43 \pm 3.21$ & NS \\
\hline 11 AXE INCL P & $113.90 \pm 11.17^{\mathrm{ac}}$ & $125.83 \pm 5.77^{b}$ & $115.13 \pm 8.79 c$ & $0.012^{*}$ \\
\hline $21 \mathrm{MD}$ INCL & $2.86 \pm 1.95$ & $2.33 \pm 1.86$ & $2.47 \pm 1.80$ & NS \\
\hline $22 \mathrm{MD}$ INCL & $3.50 \pm 3.66$ & $3.66 \pm 2.58$ & $3.73 \pm 2.33$ & NS \\
\hline 21 Axis INCL p & $113.13 \pm 12.22^{\mathrm{ac}}$ & $124.50 \pm 6.65^{\mathrm{b}}$ & $114.60 \pm 7.46^{c}$ & $0.029 *$ \\
\hline $31 \mathrm{MD}$ INCL & $4.09 \pm 3.44$ & $2.00 \pm 1.78$ & $3.30 \pm 2.61$ & NS \\
\hline $32 \mathrm{MD}$ INCL & $5.72 \pm 4.72$ & $6.33 \pm 4.41$ & $5.04 \pm 3.06$ & NS \\
\hline 31 Axis INCL m & $99.40 \pm 9.44^{a}$ & $109.66 \pm 10.21^{b}$ & $97.26 \pm 8.19$ ac & $0.034^{*}$ \\
\hline $41 \mathrm{MD}$ INCL & $3.59 \pm 3.12$ & $3.83 \pm 2.78$ & $2.43 \pm 2.21$ & NS \\
\hline $42 \mathrm{MD}$ INCL & $6.31 \pm 5.76$ & $6.50 \pm 6.25$ & $5.56 \pm 4.11$ & NS \\
\hline 41 Axis INCL m & $97.63 \pm 7.32^{a}$ & $107.83 \pm 7.22^{\mathrm{b}}$ & $97.30 \pm 8.95^{\mathrm{ac}}$ & $0.025^{*}$ \\
\hline $\mathrm{l} / \mathrm{i}$ & $112 \pm 11.60^{\mathrm{ab}}$ & $104.66 \pm 4.45^{b}$ & $109.78 \pm 8.47^{c}$ & $0.014^{*}$ \\
\hline
\end{tabular}

*: indicates statistically significant differences at $\mathrm{p}<0.05$; NS= not significant. Kruskal Wallis $\mathrm{H}$ test with Post-hoc comparison $a, b, c$ : letters with identical exponents indicate the absence of a statistically significant difference

Table 3: Comparison of the alveolar bone thickness $(\mathrm{mm})$ of teeth between the different facial types and the results of the multiple comparison test

\begin{tabular}{|c|c|c|c|c|}
\hline Alveolar bone thickness & Normodivergent $(n=22)$ & Hypodivergent $(n=6)$ & Hyperdivergent $(n=23)$ & Kruskal Wallis ( $p$-value) \\
\hline 11 Max CLP & $2.21 \pm 1.36^{a}$ & $1.77 \pm 1.03^{\mathrm{abc}}$ & $3.48 \pm 2.39^{c}$ & $0.047^{*}$ \\
\hline 22 Max DLP & $7.63 \pm 1.42^{\mathrm{abc}}$ & $4.50 \pm 2.59 \mathrm{~b}$ & $9.00 \pm 3.15^{c}$ & $0.008^{*}$ \\
\hline 32 Man DLL & $4.03 \pm 2.24^{a}$ & $3.12 \pm 2.02^{\mathrm{ab}}$ & $5.46 \pm 2.62^{c}$ & $0.043^{*}$ \\
\hline 41 Man DLL & $3.87 \pm 2.05^{a}$ & $2.55 \pm 1.20^{\mathrm{ab}}$ & $4.63 \pm 1.95^{c}$ & $0.014^{*}$ \\
\hline $16 \mathrm{Max}$ DLV & $1.67 \pm 1.58^{\mathrm{a}}$ & $1.90 \pm 1.25^{\mathrm{abc}}$ & $3.71 \pm 2.24^{c}$ & $0.002^{*}$ \\
\hline 26 Max DLV & $2.36 \pm 1.74^{a}$ & $1.90 \pm 1.35^{\mathrm{ab}}$ & $3.68 \pm 1.93^{c}$ & $0.022^{*}$ \\
\hline 26 Max DLP & $2.21 \pm 1.61^{a}$ & $1.90 \pm 1.54^{\mathrm{ab}}$ & $3.67 \pm 1.89 c$ & $0.013^{*}$ \\
\hline 36 Man DLL & $8.35 \pm 2.97^{a}$ & $6.89 \pm 3.51^{\mathrm{ab}}$ & $11.47 \pm 3.46^{c}$ & $0.004^{*}$ \\
\hline 46 Man DLL & $8.77 \pm 3.54^{\mathrm{abc}}$ & $6.45 \pm 2.43^{b}$ & $10.67 \pm 3.86^{c}$ & $0.041^{*}$ \\
\hline
\end{tabular}

*: indicates statistically significant differences at $p<0.05 ; \mathrm{NS}=$ not significant

$a, b, c:$ letters with identical exponents indicate the absence of a statistically significant difference

\section{DISCUSSION}

According to the results of our study, the teeth are tilted differently according to the vertical skeletal typology and the corresponding alveolar bones do not always have the same thickness. The varied inclinations were more pronounced in the anterior sector. Indeed, hypodivergent subjects showed a greater vestibular inclination of the maxillary and mandibular incisors compared to normal or hyper-divergent subjects.

Comparison of the results of our study with those of previous studies should be done with caution due to differences in sample selection and measurement techniques used.

Like Ross et $a{ }^{5}{ }^{5}$ we found no statistically significant difference in the inclination of the first molars between the 3 different growth profile subgroups.

The absence of statistically significant differences could be due either to the limitations of the facial index used, which may not have chosen very extreme face types, or to the relatively small number of subjects in the horizontal pattern groups $(n=6)$.

However, we found a greater tendency to lingual inclination in hyperdivergent subjects compared to horizontally growing subjects. This trend was observed by Grosso et al. ${ }^{7}$ These authors had small groups for both short and long face types. Perhaps these represented the extremes of each type, and therefore a difference was highlighted.

The anterior area is more demanding in terms of aesthetics and treatment according to bone availability. ${ }^{10}$

In any case, the lack of optimal bone availability around the teeth can be responsible for recurrence after orthodontic treatment. The $110.13^{\circ} \pm 9.76$ inclination of the interincisal angle of our sample was much smaller than the interincisal angle of $131^{\circ}$ reported in the literature." This can be explained by the fact that 
the subjects in our sample were all black Africans and therefore in bimaxillary dentoalveolar biprotrusion.

Using $\mathrm{CBCT}$, the second finding of this study is that, in general, the bone wall is thicker in the lingual than in the vestibular, regardless of the vertical skeletal typology. This observation was relayed by Lee et $\mathrm{al}^{12}$ in 2010.

However, these results are different from those reported in studies by Horner et $\mathrm{al}^{13}$ which showed that vestibular cortical bone tends to be thicker in hypodivergent patients than in hyperdivergent patients. This discrepancy with the results of our study could be attributed to the small number of selected hypodivergent.

When dental orthodontic movements are planned, the position and inclination of the teeth, as well as the thickness of the alveolar bone, should be considered as "diagnosis" variables. ${ }^{14}$ In our study, we observed that the thickness of the vestibular alveolar bone at the apex was thinner than at the lingual side in hyperdivergent patients. This result is consistent with Eraydin's. ${ }^{15}$

In general, a thorough assessment of the alveolar bone thickness of the teeth is necessary to determine the limits of possible dental movement during orthodontic treatment. Dental displacement is the basis of orthodontic therapy. It is the result between the application of a force to a tooth and the strength of its supporting tissues. Knowledge of alveolar bone volume can help orthodontists to better control the forces applied to avoid iatrogenic bone loss and bone fenestration.

\section{CONCLUSION}

Dental inclinations are closely related to the thickness of the alveolar bone associated with them. There is a significant association between bone thickness around the teeth and facial profile type. The CBCT provides clear images of incisor and molar regions and alveolar spaces in different planes, which can help orthodontists to better control the applied forces.

The morphology of the alveolar bone of the lower central incisor may be affected by the inclination of the incisor. The apex of the root of the lower central incisor is closer to the lingual alveolar crest when it was inclined lingually. These different dental positions are important and must be taken into account in the orthodontic treatment plan.

\section{CONFLICTS OF INTEREST}

No potential conflict of interest relevant to this article was reported.

\section{OJN}

\section{REFERENCES}

1. Tsunori M, Mashita M, Kasay K. Relationship between facial types and tooth and bone characteristics of the mandible obtained by CT scanning. Angle Orthod 1998; 68(6):557-62

2. Gracco A, Luca L, Bongiorno MC, Siciliani G. Computed tomography evaluation of mandibular incisor bony support in untreated patients. Am J Orthod Dentofacial Orthop 2010; 138(2):179-187.

3. Andrews $L$ The six keys to normal occlusion. Am J Orthod 1972; 62(3):296-30921.

4. $\mathrm{X} \cup \mathrm{H}, \mathrm{Han} X$, Wang $Y$, Shu $R$, Jing $Y$, Tian $Y$, et al. Effect of buccolingual inclinations of maxillary canines and premolars on perceived smile attractiveness. Am J Orthod Dentofacial Orthop. 2015;147(2):182-189.

5. Ross VA, Isaacson RJ, Germane N, Rubenstein LK. Influence of vertical growth pattern on faciolingual inclinations and treatment mechanics. Am J Orthod Dentofacial Orthop 1990; 98(5):422-9.

6. Masumoto T, Hayashi I, Kawamura A, Tanaka K, Kasai K. Relationships among facial type, buccolingual molar inclination, and cortical bone thickness of the mandible. Eur J Orthod 2001;23(1):15-23.

7. Grosso LE, Rutledge M, Rinchuse DJ, Smith D, Zullo T. Buccolingual inclinations of maxillary and mandibular first molars in relation to facial pattern. Orthod Pract 2012; 5:43-48.

8. Eraydin F, Cakan DG, Tozlu M, Ozdemir F. Evaluation of buccolingual molar inclinations among different vertical facial types. Korean $J$ Orthod 2018; 48(5):333-338

9. Horn A.J. Facial height index. Am J Orthod 1992; 102(2):180-186.

10. Januário AL, Duarte WR, Barriviera M, Mesti JC, Araújo MG, Lindhe J. Dimension of the facial bone wall in the anterior maxilla: a cone-beam computed tomography study. Clin Oral Implants Res. 2011,22(10):1168-1171.

11. Steiner CC. Cephalometrics in clinical practice. Angle Orthod 1959; 29(1):8-29.

12. Lee SL, Kim HJ, Son MK, Chung CH. Anthropometric analysis of maxillary anterior buccal bone of korean adults using Cone-Beam CT. J Adv Prosthodont. 2010, 2(3):92-96.

13. Horner KA, Behrents RG, Kim KB, Buschang PH. Cortical bone and ridge thickness of hyperdivergent and hypodivergent adults. Am J Orthod Dentofacial Orthop. 2012,142(2):170-178.

14. Borzabadi-Farahani A. A review of the oral health-related evidence that supports the orthodontic treatment need indices. Prog Orthod 2012; 13(3):314-325.

15. Eraydin F, Germec-Cakan D, Tozlu M, Ozdemir Fl. Three-dimensional evaluation of alveolar bone thickness of mandibular anterior teeth in different dentofacial types. Niger J Clin Pract 2018; 21 (4):519-524. 